\title{
Framework for Client-Server Distributed Database System for Federal Road Safety Commission Nigeria
}

\author{
Sesetuperekiye E. \\ Department of Computer Science, \\ Bayelsa State Polytechnic, Aleibiri.
}

\begin{abstract}
In recent years it has been observed that the federal road safety commission FRSC has been having some challenges on how to handle traffic rule and traffic offences committed by road users both commercial and private. Based on the in ability of the FRSC to handle these problems this paper critically looked into the behavioural pattern of the drivers towards traffic rules and offences committed and found that there was a great need for the Organisation to have a good database, that will be able to keep records of traffic offences and offenders, which can be called upon at any time on reference basis. The aim of this study is to create a distributed Federal Road Safety commission traffic offence system that can access data from any state of the Federation. The system consists of a relational database of FRSC variables which could be shared by the various States and Local Government Areas in the country. Each of the local government area will form a website, and the database will be hosted by the server at the Federal Road Safety Commission Head Quarters at Abuja and at the various state capital. . All LGAs will access the database via a distributed network. The client/server distributed network architecture is used in the design and implementation of the system. The system is capable of monitoring all road offences and traffic offenders records at all levelsfrom any part of the country and generation of reports concerning offenders and also access information from the local government at all times.
\end{abstract}

Key-Words: Client, Sever, Distributed Database, Traffic, Offences, Network, Framework, Road User, System, Information, Computerized, Local Government Area (L.G.A).

\section{INTRODUCTION}

A computerized traffic offence system is a data based program which keeps record of the offences committed by road users in Bayelsa State, Nigeria. The traffic offence system helps the Federal Road Safety Commission to keep adequate and accurate record of all traffic offences committed by drivers and road users and also help to maintain the database of the commission.

A distributed computer system consist of a collection of autonomous computers linked by a computer network, and equipped with the necessary computer networking software's such as servers and browsers to effect its communication with other computers (www.answers.com). 
Navathe (2000) defined distributed database (DDB) as a collection of multiple logically integrated databases distributed over a computer network. In addition, he defined a distributed database management system (DDBMS) as a software system that manages a distributed database while making the distribution transparent to the user. The computer systems are connected together for the purpose of sharing data and information via sending message over a communication network to achieve a common objective. In this research, an attempt is made to design a framework for a client/server distributed database system for the Federal Road Safety Commission (FRSC) or ministries and local government areas of Bayelsa state.

The system consists of a relational database of FRSC variables (entities) that could be stored by the local office of the state. Each of the state office will form a website, and the database will be hosted by the headquarters of Federal Road Safety Commission office in Abuja.. The state offices will access the database via the distributed network. The client/server distributed network architecture is used for the development of the system. The system is capable of monitoring all traffic offences committed by road users in Bayelsa State.

\section{BACKGROUND OF STUDY}

The Federal Road Safety Commission of Nigeria FRSC, established by the FRSC, established by the FRSC Act Cap 14 Law of the federation of Nigeria (LFN), in February 1988, is the body that manages all traffic activities that is being carried out in Bayelsa State, Nigeria prior to its establishment, there was no concrete and sustained policy in place to manage the rate of death and accidents on Nigerian roads.

According to Ibrahim and Olukoju (2003) the commission was charged with the responsibility of reducing to the bearest minimum the consequent human and material loss and road traffic accident. With regards to this responsibility the FRSC instituted a harmonization of the various road traffic laws into the National Road Traffic Regulations (NRTR 1997). Among these laws were the highway (motor traffic) ordinance of 1913. In southern Nigeria, was Nigeria first transport law, others were the motor traffic ordinance of 1916 of nationwide applicability, the road traffic act the Federal Highway Act and the Law of carriage.

Up to late 1970's, there was little or no concern about road safety matters in Nigeria and so little or no significance was accorded to road traffic accident, prevention strategies and remedies (Wakawa and Oyeyemi:2003), despite the danger signals visible in available road crash data. Around the world road traffic injuries are a major public health challenges that requires concerted efforts for effective and sustained prevention. An estimated 1.3 million people die in road crashes around the world and sixty-five percent of these death in road crashes involve pedestrians and 35\% of pedestrian's death are to the children. Over 30-50 million injuries each year most of these injuries lead to lifelong disability including brain and spinal cord injury.

It is believed by World Health Organization (W.H.O) that the figure may or could increase by more than half over the next 20 years unless there is a firm commitment to road safety and accident prevention, it sis a dead fact that many of these accident and the after effect would have been avoided. However the computerized traffic offence system will help keep records of all traffic offence committed and will be committed by road user and also maintain the database of the commission. 
Sesetuperekiye, E. (2020) Framework for Client-Server Distributed Database System for Federal Road Safety Commission Nigeria. Advances in Social Sciences Research Journal, 7(5) 440-451.

\section{THEORY OF DISTRIBUTED DATABASE AND DISTRIBUTED DATABASE MANAGEMENT SYSTEM}

A distributed system is defined as an information processing system that contains a number of independent computers that cooperate with one another over a communication network in order to achieve a specific objective. It can also be defined as a database in which portions of the database are stored on a multiple computers within a network. The definitions above means that a distributed system is not stored in its entirety at a single location. Instead, it is spread across a network of computers that are geographically dispersed and connected through a communication network by which each site is a database of its own. The sites have agreed to work together so that data user can access data anywhere in the network exactly as if the data where all stored at the user's own site. Users have access to the portion of the database at their location so that they can access the data relevant to their tasks without interfering with the work of others. Distributed databases system use a client/server architecture to process information requests. It allows faster local queries and can reduce network traffic. The diagram below illustrates it all

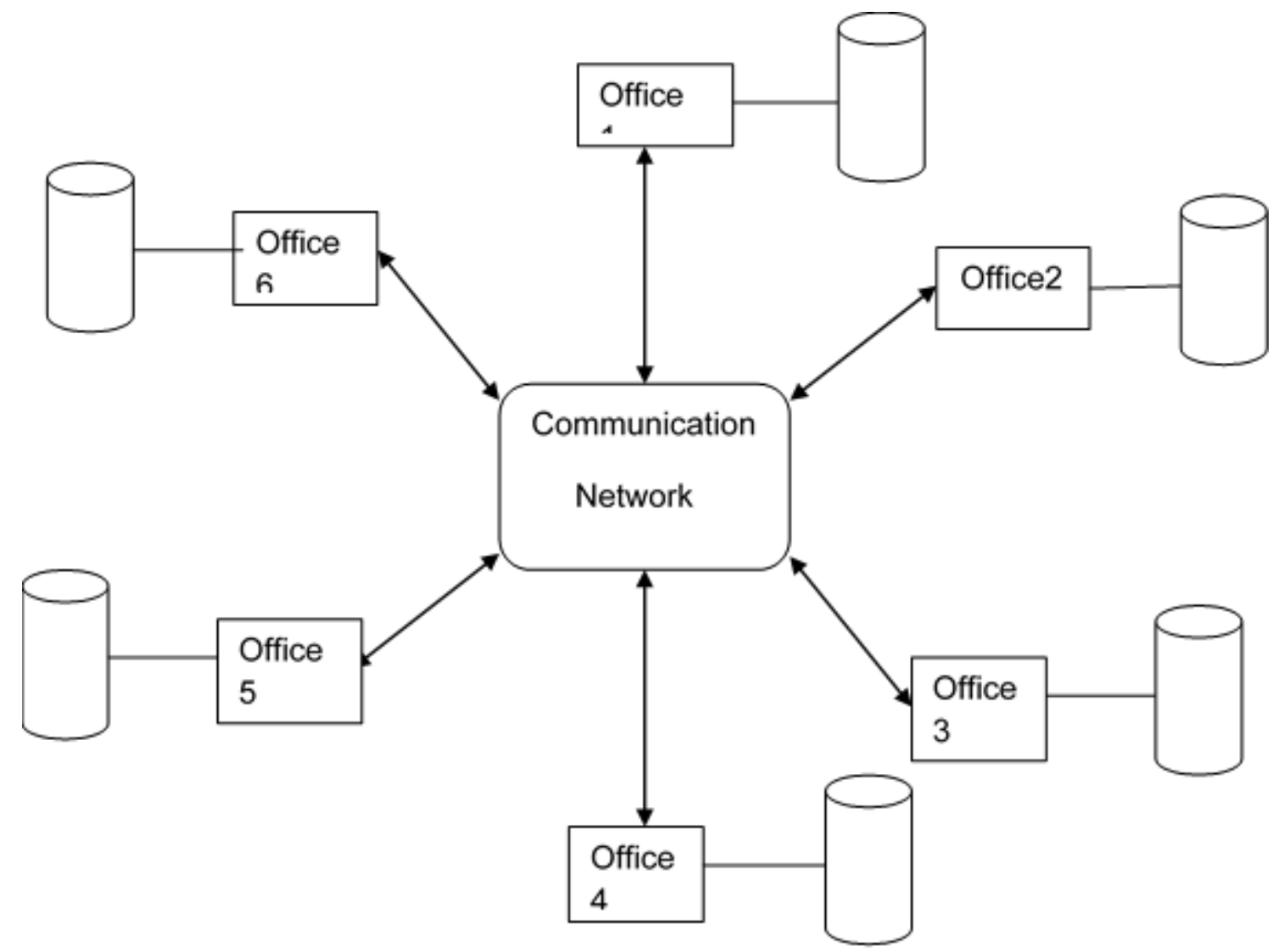

Figure 1. A Distributed Database Architecture

\section{Advantages of Distributed Database System}

- Management of distributed data is done with different levels of transparency (Replication , Fragmentation, Location, Performance, Transaction and Catalog transparency.

- Capacity and Growth: An advantage of distributed databases is that as the organization grows, new sites can be added with little or no upheaval to the DBMS. This situation can be 
compared to a centralized system, where growth entails upgrading with changes in hardware and software that affect the entire database.

- Reliability and Availability: An advantage of distributed database is that even when a portion of a system (i.e. a local site) is down, the overall system remains available. With replicated data, the failure of one site still allows access to the replicated copy of the data from another site. The remaining sites continue to function. The greater the accessibility the more it enhances the reliability of the system.

- Efficiency and Flexibility: An advantage of distributed databases is that data is physically stored close to the anticipated point of use. Hence, if usage pattern changes, then data can be dynamically moved or replicated to where it is most needed.

- Distributed Database Sharing: An advantage of distributed databases is that users at a given site are able to access data stored at other sites and at the same time retain control over the data at their own site.

- Protection of Valuable Data: Assuming there was a catastrophic event such as a fire outbreak, all of the data would not be in one place, but distributed in multiple locations.

- Improved Performance: Data is located near the site of greatest demand, and the database systems themselves are parallelized, allowing load on the databases to be balanced among servers (A high load on one module of the database would not affect other modules of the database in a distributed database).

\section{Disadvantages of Distributed Database System}

- Increased storage and infrastructure requirements because multiple copies of data are required at various separate locations which would require more disk space.

- Security lapses have increased since data are in multiple locations.

- Integrity control becomes more difficult.

- Database design becomes more complex.

A key objective for a distributed system is that it looks like a centralized system to the user. The user does not need to know where a piece of data is stored physically.

\section{Categories of Distributed Data}

There are five categories of distributed data. They include:

- Replicated data

- Horizontally fragmented data

- Vertically fragmented data

- Reorganized data

- Separate-schema data

A distributed database management system (DDBMS) is a software system that permits the management of a distributed database and makes the distribution transparent to the users. A centralized distributed database management system (DDBMS) manages the database as if it were all stored on the same computer. The DDBMS synchronizes all the data periodically, and in cases where multiple users must access the same data, it ensures that updates and deletes performed on the data at one location will be automatically reflected in the data stored elsewhere. The users and administrators of a distributed system should with proper implementation, interact with the system 
Sesetuperekiye, E. (2020) Framework for Client-Server Distributed Database System for Federal Road Safety Commission Nigeria. Advances in Social Sciences Research Journal, 7(5) 440-451.

as if the system was centralized. Query optimization is essential if a DBMS is to achieve acceptable performance and efficiency. Distributed Database Management System is required to maintain distributed database and make it transparent to clients. Sometimes, distributed database is used to refer jointly to the distributed database management system. Consequently, an application can simultaneously access and modify the data in several databases in a network. The main thing that all of such systems have in common is the fact that data and software are distributed over multiple sites connected by some form of communication network. However, the Distributed Database Management System basically addresses the following technical processes:

- Replica Synchronization: This is about synchronizing data based on relatively smaller transactions where the said transactions may consist of several read and write operations on the server. But some applications can take relatively bigger data production jobs. Also, it can write a whole file which can be a relatively large transactional file.

- Synchronous and Asynchronous Replication: Replication may be done through synchronous or asynchronous or batch replication method which makes replicas be in synch (synchronous) or out of sync (asynchronous) for a certain period of time. Update reconciliation may be done at certain time intervals such as every hour or every night.

- Network Servers and Load: This refers to the management of computer on the network networks nodes which can act either as server or client or both server and client at certain circumstances. Under this area, other important considerations include traffic management and security aspects.

- Heterogeneous Data Stores Management: Different computer servers may be implemented on different platforms, so support for heterogeneous data store should be greatly considered. Different kinds of data may be stored in different formats by different vendors. Even in the case of two different database paradigms namely; relational and object oriented, a DDBMS needs to consider this aspect. The standard protocol used for directory information such as Lightweight Directory Access Protocol (LDAP) falls under this consideration.

\section{Client/Server}

Client/server systems are constructed so that the database can reside on a central computer, known as a server, and be shared among several users. Users access the server through a client or server application. In large client/server systems, thousands of users may be connected to a SQL Server installation at the same time. SQL Server has full protection for these environments, with safeguards that prevent problems such as having multiple users trying to update the same piece of data at the same time. SQL Server also allocates the available resources effectively, such as memory, network bandwidth, and disk input/output, among the multiple users.

Advantages of Client/Server

- More efficient division of labour

- Horizontal and vertical scaling of resources

- Better price/performance on client machines

- Ability to use familiar tools on client machines

- Client access to remote data (through standards)

- Full DBMS functionality provided to client workstations 


\section{Collaborating Server}

Here, we can have a collection of database servers, each capable of running transactions against local data, which cooperatively execute transactions spanning multiple servers. When a server receives a query that requires access to data at other servers, it generates appropriate sub-queries to be executed by other servers and puts the results together to compute answers to the original query. Ideally, the decomposition of the query should be done using cost-based optimization, taking into account the costs of network communication as well as the local processing costs.

Middleware: Middleware is a crucial component of modern IT infrastructure. It is a set of common business-unaware services that enable applications and end users to interact with each other across a network. In essence, middleware is the software that resides above the network and below the business-aware application software. The services provided by these routines are available to the applications through application programming interfaces (APIs) and to the human users through commands and/or graphical user interfaces (GUIs).

\section{FRAMEWORK FOR THE CLIENT/SERVER ARCHITECTURE OF A DISTRIBUTED SYSTEM FOR FEDERAL ROAD SAFETY COMMISSION}

Among the types of system architectures for information processing, the client-server architecture is chosen for this framework. A Client is a computer or device requesting data from the Server, which is a computer hosting the data. It works through a network protocol, more likely than TCP/IP. Servers are computers that hold the actual databases and run only the database management system and related software. They are usually multiprocessor computers, with generous memory and raid disk arrays used for stable storage. Hardware database accelerators, connected to one or more servers via a high-speed channel, are also used in large volume transaction processing environments. Clients rely on servers for resources, such as files, devices, and even processing power.

Interaction between client and server might proceed as follows during the processing of a SQL query:

- The client parses a user query and decomposes it into a number of site queries. Each site query is sent to the appropriate server site.

- Each server processes the local query and sends the resulting relation to the client site.

- The client site combines the results of the sub-queries to produce the result of the originally submitted query.

In this approach, the SQL server has also been called a transaction server (or a database processor (DP) or a back-end machine), whereas the client has been called an application processor (AP). In large client/server systems, thousands of users may be connected to a SQL Server at the same time. SQL Server has full protection for these environments, with safeguards that prevent problems such as having multiple users trying to update the same piece of data at the same time. SQL Server also effectively allocates the available resources, such as memory, network bandwidth, and disk I/O, among the multiple users. While SQL Server works effectively as a server, it can also be used in applications that need stand-alone databases stored locally on the client. SQL Server can configure itself dynamically to run efficiently with the resources available on a client, without the need to dedicate a database administrator to each client. However, the interaction between client and 
Sesetuperekiye, E. (2020) Framework for Client-Server Distributed Database System for Federal Road Safety Commission Nigeria. Advances in Social Sciences Research Journal, 7(5) 440-451.

server can be specified by the user at the client level or through a specialized DBMS client module that is part of the DBMS package. For example, the user may know what data is stored in each server; break down a query request into site sub-queries manually; and submit individual sub-queries to the various sites.

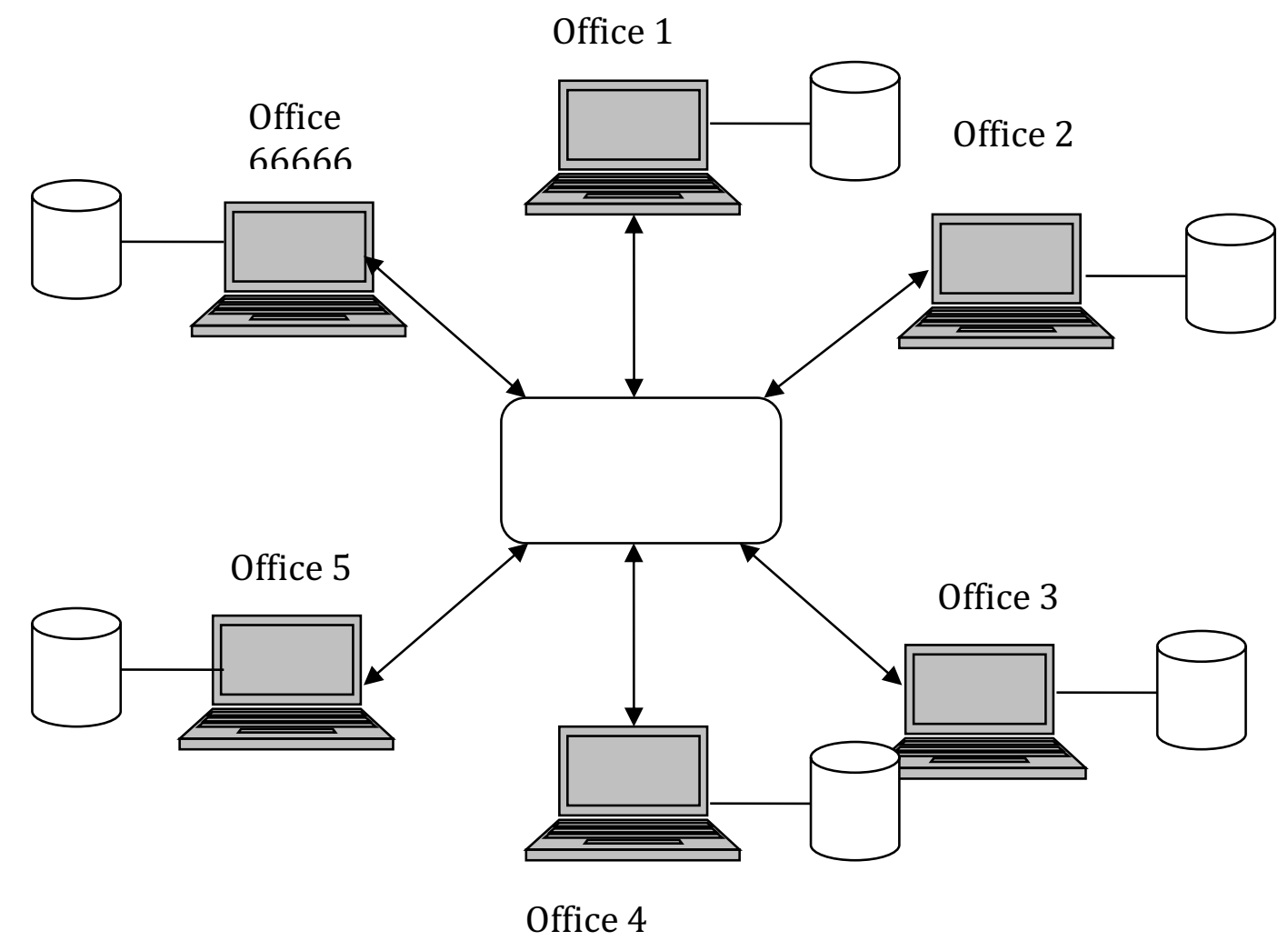

Figure 2. Client/Server Architecture of a Distributed System

Software modules in a typical DDBMS are divided into three levels:

- The server software is responsible for local data management at a site, much like the centralized DBMS software.

- The client software is responsible for most of the distribution functions; it accesses data distribution information from the DDBMS catalog and processes all requests that require access to more than one site. It also handles all user interfaces.

- The communications software (sometimes in conjunction with a distributed operating system) provides the communication primitives that are used by the client to transmit

- Commands and data among the various sites as needed. This is not strictly part of the DDBMS, but it provides essential communication primitives and services.

The client is responsible for generating a distributed execution plan for a multi-site query or transaction and for supervising distributed execution by sending commands to servers. These commands include local queries and transactions to be executed, as well as commands to transmit data to other clients or servers. Hence, client software should be included at any site where multisite queries are submitted. Another function controlled by the client (or coordinator) is that of 
ensuring consistency of replicated copies of a data item by employing distributed concurrency control techniques.

\section{SYSTEM DESIGN}

The Federal Road Safety Commission will have its design as follows.

\section{Database Design}

A database is a collection of logically related files (tables). A database management system is a software program used in the creation and management of database. A file (table) is a collection of logically related records. Each record is uniquely identified by a primary key. A record is a collection of logically related fields (columns) that uniquely identifies a person. Here is a list of information that will be required at the FRSC to show that an individual is an offender of the traffic rules.

\section{Input Design of The Offender}

Offender Registration Form: This form is used to enter information about the offender into the system's database.

\section{Upload Form Details}

\begin{tabular}{|c|c|c|}
\hline FIELD NAME & DATA TYPE & Size \\
\hline First Name & Short text & 50 \\
\hline Last Name & Short text & 50 \\
\hline Sex & Short text & 10 \\
\hline Occupation & Short text & 50 \\
\hline Address & Short text & 100 \\
\hline Name of LGA & Short text & 50 \\
\hline State & Short text & 30 \\
\hline Nationality & Short text & 20 \\
\hline Offence class & Short text & 10 \\
\hline License number & Short text & 20 \\
\hline Report Date & Date/Time & \\
\hline Reporting Marshal id & Short text & 50 \\
\hline Marshal comment & Long text & 200 \\
\hline \multicolumn{2}{|c|}{ Vehicle details } \\
\hline Var plate number & Short text & 50 \\
\hline Vehicle type & Short text & 50 \\
\hline Vehicle color & Short text & 50 \\
\hline Chassis No & Short text & 50 \\
\hline
\end{tabular}

\section{Search Result}

\begin{tabular}{|c|c|c|}
\hline FIELD NAME & DATA TYPE & SIZE \\
\hline Offender name & Short text & $\mathbf{5 0}$ \\
\hline Driver's License number & Short text & $\mathbf{2 0}$ \\
\hline
\end{tabular}

\section{Log In Form}

\begin{tabular}{|c|c|c|}
\hline FIELD NAME & DATA TYPE & SIZE \\
\hline User Name & Short text & 11 \\
\hline Password & Short text & 11 \\
\hline
\end{tabular}


Sesetuperekiye, E. (2020) Framework for Client-Server Distributed Database System for Federal Road Safety Commission Nigeria. Advances in Social Sciences Research Journal, 7(5) 440-451.

A careful study and analysis of the above data suggest that while the different sites (the offices) collect data differently and through disjointed efforts, they all basically require the same set of data. In this research, an attempt was made to formulate a standard operational procedure for the FRSC headquarters alongside with the sites (the offices), so as to bring effectiveness in detecting road traffic offenders and will be able to bring culprits to book no matter the local of where the offence is committed. Moreover, a centrally managed database is presented with an element of multi-level data access.

\section{Components Of The Distributed System}

The distributed system has Internet facing Web-enabled applications that can be accessed remotely by the users either within the organization or remotely. The following are a list of the information technology (IT) infrastructure components of the system

Firewall: A system designed to prevent unauthorized access to or from a private network. Firewalls can be implemented in both hardware and software, or a combination of both. Firewalls are frequently used to prevent unauthorized Internet users from accessing private networks connected to the Internet, especially intranets. All messages entering or leaving the intranet pass through the firewall, which examines each message and blocks those that do not meet the specified security criteria. There are several types of firewall techniques:

- Packet Filter: This looks at each packet entering or leaving the network and accepts or rejects it based on user-defined rules. Packet filtering is fairly effective and transparent to users, but it is difficult to configure. In addition, it is susceptible to Internet Protocol (IP) spoofing.

- Application Gateway: This applies security mechanisms to specific applications, such as File Transfer Protocol (FTP) and Telnet servers. This is very effective, but can impose performance degradation.

- Circuit-Level Gateway: This applies security mechanisms when a Transmission Control Protocol (TCP) or User Datagram Protocol (UDP) connection is established. Once the connection has been made, packets can flow between the hosts without further checking.

- Proxy Server: Intercepts all messages entering and leaving the network. The proxy server effectively hides the true network addresses.

- Router: A router is a special purpose computer or software device that enables two or more dissimilar networks to communicate. Routers route traffic, which consists of Transmission Control Protocol/Internet Protocol (TCP/IP) packets.

- Host: A computer that is connected to a TCP/IP network, including the Internet.

- Server: A server is a dedicated computer that allows other computers to connect to it.

The following are the various types of sever available:

- Domain Name System

- Web servers

- Internet banking servers

- E-mail servers

- Proxy servers

Workstations: In networking, a workstation refers to any computer connected to a local area network. It could be a workstation or a personal computer. 
Intrusion Detection Systems: Intrusion detection is fundamentally the process of monitoring computer networks and systems for violations of computer policy.

\section{Network Structure}

A metropolitan area network (MAN), provides long distance transmission of data, image, audio, video information over large geographic area that may comprise of a country, a continent, or even the whole world. MAN is best for this kind of system. This system grew from earlier community antenna systems used in areas with poor over-the-air television reception. In these early systems, a large antenna was placed on top of a nearby hill and the signal was then piped to the subscriber's homes. The diagram below describes the structure of this framework.

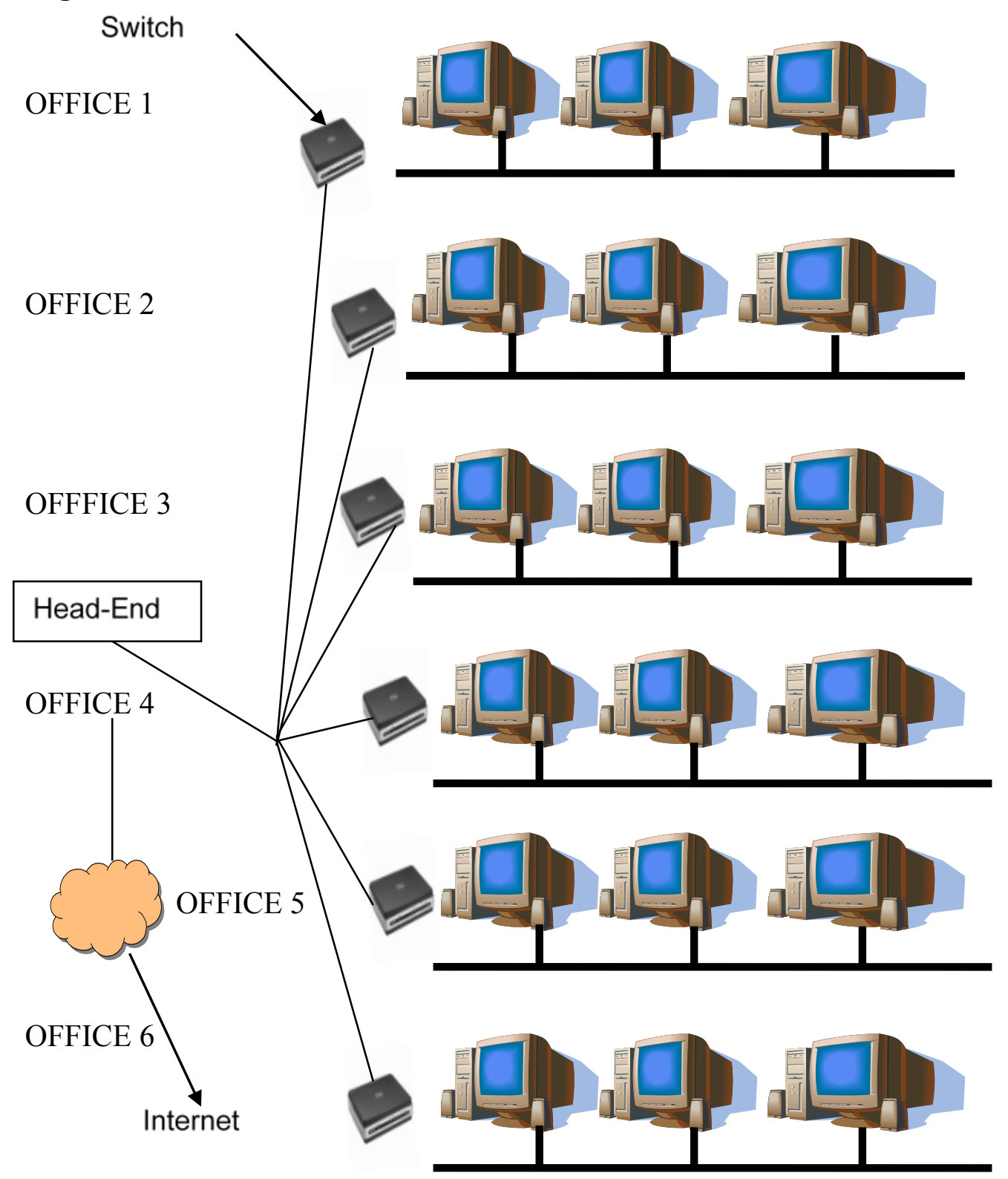

Fig 5.1 Metropolitan Area Network (MAN) based system 


\section{CONCLUSION}

The use of a distributed database management system (DDBMS) by the Federal Road Safety Commission is a reliable, secured, and effective way of monitoringtraffic offenders. The system has removed delays which were experienced through the old system because cross checking through the traffic records has been time consuming. The traffic law enforcers has benefited from the new system because there are no longer delays in storing and retrieving traffic offences. This stems from the fact that the management of distributed data is done with different levels of transparency. However, such transparency include: replication transparency, fragmentation transparency, location transparency, performance transparency, transaction transparency, and catalog transparency. A distributed database gives an added advantage for new sites to be added with little or no upheaval to the DBMS as the organization grows and expands.

Furthermore, employees data stored are reliable and easily accessible. For instance, when a part of the system (i.e. a local site) is down, the overall system still remains available. With replicated data, the failure of one site still allows access to the replicated copy of the data from another site. Thus, the remaining sites continue to function. It is efficient, flexible, and supports file sharing. As a result, users at a given site are able to access data stored at other sites and at the same time retain control over the data at their own site. The use of a distributed database for the federal road safety commission helps to maintain high level of security and protection ofoffenders and employee's data in the various local government and states. Assuming there was a catastrophic event such as a fire outbreak, all of the data would not be in one place, but distributed in multiple locations. As such, this makes it possible to have access to same data from other location. It has a firewall which helps to prevent unauthorized access to or from a private network. This fire wall might be in the form of packet filter, application gateway, circuit-level gateway, or a proxy server. Other components of the distributed network system that helps to maintain high level of security include a router, host, server, workstation, and an intrusion detection system.

Consequently, the use of a client-server distributed database system for Federal Road Safety Commission shows an improved performance. Data is located near the site of greatest demand, and the database systems themselves are parallelized, allowing load on the databases to be balanced among servers. This distributed database management system employs a metropolitan area network (MAN). The Metropolitan Area Network (MAN) provides long distance transmission of data, image, audio, video information over large geographic area that may comprise of a country, a continent, or even the whole world. In conclusion, the use of the client-server distributed database for an internal revenue system would facilitate a secured, reliable, and available system for keeping records of internally generated revenues as well as financial records of employee's in the different local government areas in Balyesa State, Nigeria.

\section{LIMITATIONS AND FURTHER RESEARCH}

Despite all its advantages, there are some limitations as to which the system can operate and these includes;

- Increased storage and infrastructure requirements because multiple copies of data are required at various separate locations which would require more disk space.

- Security lapses have increased since data are in multiple locations. 
- Integrity control becomes more difficult.

- Database design becomes more complex.

Based on these limitation I can say further research can be made on each these limitations as to encourage further studies.

\section{References}

Blumenthal, S.C (1999). Management Information System; New Jersey: Hall Inc. Eaglewood Publishers.

Brutus, L. (1991). A Database Management Information System; India: Division of Macmillan publishing.

Candace .C. Fleming and Barbara von Halle (1989): Handbook of Relational Database Design. Addison Wesley

Longman.

Charles P. Pfleeger and Shari Lawrence Pfleeger (2003): Security in Computing,

David M.A (1999). FRSC and the Imperatives of Road Traffic Safety in Nigeria.Jos: Chohu Business Enterprise

Elmasri and Navathe, Fundamentals of database systems, 3rd Edition. Addison-Wesley Longman.

Federal Road Safety Commission Nigeria (2008).Nigeria Highway Code;

Abuja: Detail woks Ltd.

Federal Republic of Nigeria (2004).National Road Traffic Regulations; Lagos: Federal Government Press.

Federal Republic of Nigeria (1949).Chapter 548. Road Traffic Act; Lagos: Federal Government Press.

Federal Republic of Nigeria (1988).Decree No.45, 1988.http://www.FRSC.gov.ng/History.html

Federal Road Safety Commission, Website. http://www.frsc.gov.ng.2016.

Fred R. McFadden and Jeffrey A. Hoffer(1994): Modern Database management. England. Addison Wesley Longman http://www.cs.rpi.edu/ noel/distr_scaleup/distriuted.html, May 17, 2004.

Navathe, E. (2000): Fundamental of Database Systems, 3rd Edition.Teturo Sawada Exclusive Publisher and Distributor Ozsu, M. T. and Valduriez, P. (2011): Principles of Distributed Database System, 3rd Edition. Springer.

Prentice Hall Professional Technical Reference, Upper Saddle River, New Jersey.

Rick Noel, “Scale Up in Distributed Databases: A Key Design Goal for Distributed System,"

The American Heritage ${ }^{\circledR}$ New Dictionary of Cultural Literacy, Third Edition

Copyright (C) 2005 by Houghton Mifflin Company.Published by Houghton Mifflin Company

Thomas M. Connolly and Carolyn E. Begg (2002): Database Systems (A Practical Approach to Design, Implementation, and Management. Third Edition. Pearson Education Limited. Addison Wesley.

Thinking Beyond Borders: Management of Extended Business Travelers (2012), KPMG International Cooperative ("KPMG International"). 